ARTICLE

DOI: $10.1038 / \mathrm{s} 41467-018-05986-9$

\title{
Controlling electric potential to inhibit solid- electrolyte interphase formation on nanowire anodes for ultrafast lithium-ion batteries
}

Won Jun Chang ${ }^{1}$, Su Han Kim¹, Jiseon Hwang ${ }^{2}$, Jinho Chang ${ }^{2}$, Dong won Yang ${ }^{1}$, Sun Sang Kwon ${ }^{1}, J_{i n}$ Tae Kim $^{1}$ Won Woo Lee ${ }^{1}$, Jae Hyung Lee ${ }^{1}$, Hyunjung Park ${ }^{3}$, Taeseup Song $\mathbb{1}^{3}{ }^{3}$, In-Hwan Lee $^{4}$, Dongmok Whang ${ }^{4} \&$ Won II Park (1) 1,5

With increasing demand for high-capacity and rapidly rechargeable anodes, problems associated with unstable evolution of a solid-electrolyte interphase on the active anode surface become more detrimental. Here, we report the near fatigue-free, ultrafast, and highpower operations of lithium-ion battery anodes employing silicide nanowires anchored selectively to the inner surface of graphene-based micro-tubular conducting electrodes. This design electrically shields the electrolyte inside the electrode from an external potential load, eliminating the driving force that generates the solid-electrolyte interphase on the nanowire surface. Owing to this electric control, a solid-electrolyte interphase develops firmly on the outer surface of the graphene, while solid-electrolyte interphase-free nanowires enable fast electronic and ionic transport, as well as strain relaxation over 2000 cycles, with $84 \%$ capacity retention even at ultrafast cycling ( $>20 \mathrm{C}$ ). Moreover, these anodes exhibit unprecedentedly high rate capabilities with capacity retention higher than $88 \%$ at $80 \mathrm{C}$ (vs. the capacity at 1C).

\footnotetext{
${ }^{1}$ Division of Materials Science and Engineering, Hanyang University, Seoul 04763, Republic of Korea. ${ }^{2}$ Department of Chemistry and Research Institute for Convergence of Basic Sciences, Hanyang University, Seoul 04763, Republic of Korea. ${ }^{3}$ Department of Energy Engineering, Hanyang University, Seoul 04763, Republic of Korea. ${ }^{4}$ School of Advanced Materials Science and Engineering, Sungkyunkwan University, Suwon 16419 , Republic of Korea. ${ }^{5}$ HYU-HPSTAR-CIS High Pressure Research Center, Hanyang University, Seoul 04763, Republic of Korea. Correspondence and requests for materials should be addressed to W.P. (email: wipark@hanyang.ac.kr)
} 
$\mathrm{T}$ he keys to meeting the ever-increasing demand for highly efficient and ultrafast rechargeable batteries include establishing high-performance electrodes with sufficient energy storage capacity, structural integrity and stability, and fast electron/ion transport ${ }^{1,2}$. As an alternative to conventional graphite anodes, which have an unsatisfactory capacity of $372 \mathrm{mAh} \mathrm{g}^{-1}$, higher capacity materials (e.g., $\mathrm{Si}^{3-5}, \mathrm{Ge}^{6}$, and $\mathrm{Sn}^{7}$ ) have been widely explored. However, these materials tend to exhibit substantial capacity fading with cycling, which is primarily due to their large volume change and the evolution of an unstable solid-electrolyte interphase (SEI) on the electrode surface ${ }^{8,9}$. A variety of nanostructured anodes with engineered morphologies and feature sizes can be used to effectively relax the strain caused by extensive volume change; however, this creates a trade-off with SEI stability due to the enlarged surface area of the anode exposed to the electrolyte ${ }^{10,11}$. This becomes more severe for higher capacity materials and with increased cycling rates, making fast and long cycle operation a greater challenge. Recently, to stabilize the SEI on nanostructured anodes, a novel hybrid design concept was developed. Pioneering examples of this concept were implemented by employing a clamping layer on hollow materials (e.g., $\mathrm{Si} / \mathrm{SiO} x^{12}$ nanotubes) or caging nanoscale particles with hollow shell layers (e.g., Si@ $\mathrm{C}^{13}$ and Ge@ $\mathrm{C}^{6}$ yolk-shell structures). In those structures, the sturdy shell layers provide a surface for stable SEI formation by preventing direct contact between the electrolyte and the anode, while an internal void space allows for free expansion of the anode material. These approaches led to stable operation with enhanced cycle lifetimes. Nevertheless, the presence of the shell/SEI layer over the large surface of each nanoscale core increases the series resistance for Li-ion and electron transport between the individually engineered nanostructures. This resistance is negligible at slow cycling but becomes problematic under extremely fast cycling 9 .

Given that most electrochemical reactions are governed by a potential difference or gradient, controlling the potential can be explored as an alternative to SEI engineering beyond previous approaches that focused primarily on the physical interface itself $^{6,7,12,13}$. Here, we propose an approach that prevents the formation of an SEI layer on the active anode by engineering the electric potential across the electrochemical interface. As a proofof-concept for the proposed strategy, we explore anodes with a high density of nickel silicide nanowires (NiSiNWs) anchored selectively to the inner surfaces of graphene-based micro-tubes

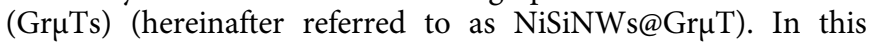
anode design, the electrolyte inside the GruTs is electrically separated from an external potential load, eliminating the buildup of potential difference that drives SEI formation on the surface of the NiSiNW anode during lithiation. As a result of this electric control, the NiSiNWs@GruT anode demonstrated excellent performance with a high specific capacity over $700 \mathrm{mAh} \mathrm{g}^{-1}$ (corresponding to $84 \%$ capacity retention), even after 2000 cycles at 20C. In addition, the capacity fade is markedly reduced with a capacity of $780 \mathrm{mAh} \mathrm{g}^{-1}$ at $80 \mathrm{C}$, retaining more than $88 \%$ of the capacity at $1 \mathrm{C}$.

\section{Results}

Strategy to engineering electric potential across the electrochemical interface. Figure 1a schematically illustrates the halfcell of the electrolyte and general nanowire (NW) anode on a current collector. Figure 1c shows the change in the structure during the first battery charging and corresponding finite element analysis (FEA) simulation results of the potential distribution across the electrolyte $\left(V_{\mathrm{E}}\right)$ and $\mathrm{NW}$ anode $\left(V_{\mathrm{A}}\right)$ (see also Supplementary Fig. 1). At an early stage of charging, a thin electrical double layer (EDL) develops in the organic electrolyte near the working electrode; most of the potential gradient is created in this area (left and middle panels in Fig. 1c and Supplementary Fig. 1b $)^{14}$. If the working potential of anode $\left(\sim V_{\mathrm{A}}\right)$ becomes substantially lower than the potential of SEI formation $\left(V_{\text {SEI }}\right)$, electrons are transported from the anode to the electrolyte and occupy the lowest unoccupied molecular orbital (LUMO) level (middle panel in Fig. 1c and Supplementary Fig. 1c). This process facilitates the reductive decomposition of the electrolyte and the subsequent growth of an SEI layer on the solid surface of the anode $^{2,15}$. When the $V_{\mathrm{A}}$ further decreases below the potential of lithiation $\left(V_{\text {Lith }}\right)$, regular lithiation and concurrent volume expansion occur in the NW anode (right panel in Fig. 1c and Supplementary Fig. 1d) ${ }^{16}$. The SEI on the NW surface also expands and then contracts to its original size upon delithiation. During cycling, with cyclic expansion and contraction of the NWs, the SEI undergoes repetitive breakage and regeneration. This leads to a continuous accumulation of the SEI layer, consumption of the electrolyte, and failure of the anode; this is the primary cause of capacity fading with an increased number of cycles 9 .

To overcome the fundamental limits caused by the SEI, we propose a new design concept introducing a thin wall of a potential sheath (PS) that spatially and electrically separates the electrolyte. Additionally, a high density of NW anodes is anchored to the inside face of the sheath (Fig. 1b). The PS is assumed to be sufficiently thin and lithium (Li)-permeable at $V_{\mathrm{A}}<V_{\mathrm{Lith}}$ but electrically conductive so as to possess the functionality of a conducting electrode and an electric shield. In such a scenario, the enclosed space is potentially isolated, in which the potentials of the electrode and electrolyte remain equal (i.e., $V_{\mathrm{A}}=V_{\mathrm{E}}^{*}$ in thermodynamic equilibrium) regardless of the external bias (left panel in Fig. 1d) ${ }^{17}$. Given that the SEI formation potential across the inner electrolyte and anode interface $\left(V_{\mathrm{SEI}}^{*}\right)$, is determined with respect to $V_{\mathrm{E}}^{*}$ (or LUMO is determined with respect to Fermi level in the electrolyte), $V_{\mathrm{SEI}}^{*}$ remains lower than $V_{\mathrm{A}}$ (middle and right panels in Fig. 1d). This consideration illustrates that, even at a low working potential of the anode $\left(V_{\mathrm{A}}<V_{\mathrm{Lith}}\right)$, SEI formation on the surface of the NW anode is substantially suppressed. Control experiments support this assertion (Supplementary Figs. 2, 3). As a result of these conditions, an SEI layer develops only on the outer surface region of the PS, where an abrupt potential gradient occurs ${ }^{2}$. The NWs with an SEI-free surface tolerate the large volume change and eliminate the issues related to the SEI (e.g., instability of the SEI and resistance to ion transport) ${ }^{9}$. Although the insertion and extraction of $\mathrm{Li}$ ions do not occur via the large area of NW and inner electrolyte interface, the SEI-free NW surface provides a pathway for facile Li transport as suitable for ultrafast (de) lithiation (the details are discussed later).

Anode based on silicide nanowires anchored inside graphene micro-tubes. To test the validity of our proposed concept, we explore anodes with a high density of NWs anchored selectively to the inner surface of a graphene PS with a continuously interconnected, three-dimensional micro-tubular structure (i.e., Gr $\mu \mathrm{T}$ ), as shown schematically in Fig. 2a. Multilayer graphene with this structure is well-suited for this purpose because it can function effectively as an atomically thin, flexible, robust support with excellent electrical conductivity and electric shielding characteristics. In addition, $\mathrm{Li}$ ions/atoms are permeable to the multilayer graphene through atomic defects ${ }^{18,19}$. However, the rate of ion permeation through the Gr $\mu \mathrm{T}$ is very slow with regard to the ionic conduction in the electrolyte so that the inner and external electrolytes would be electrically separated. The FEA simulation results showed that more than $97 \%$ of the potential gradient 
a

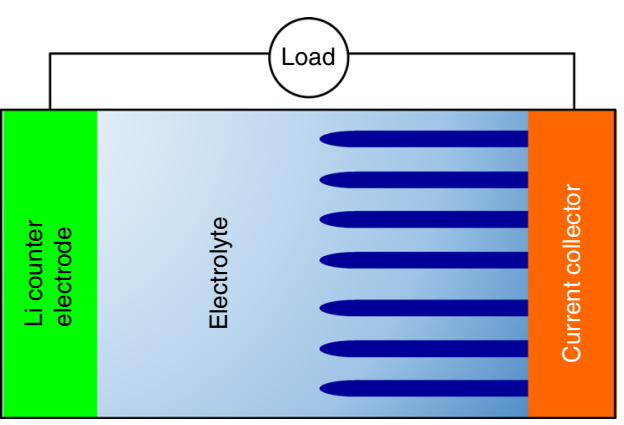

b

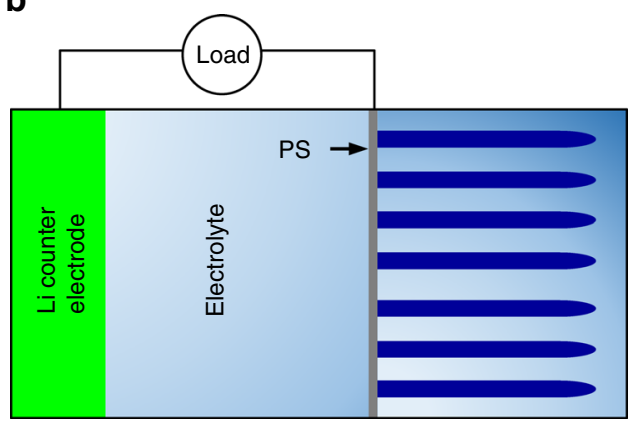

C
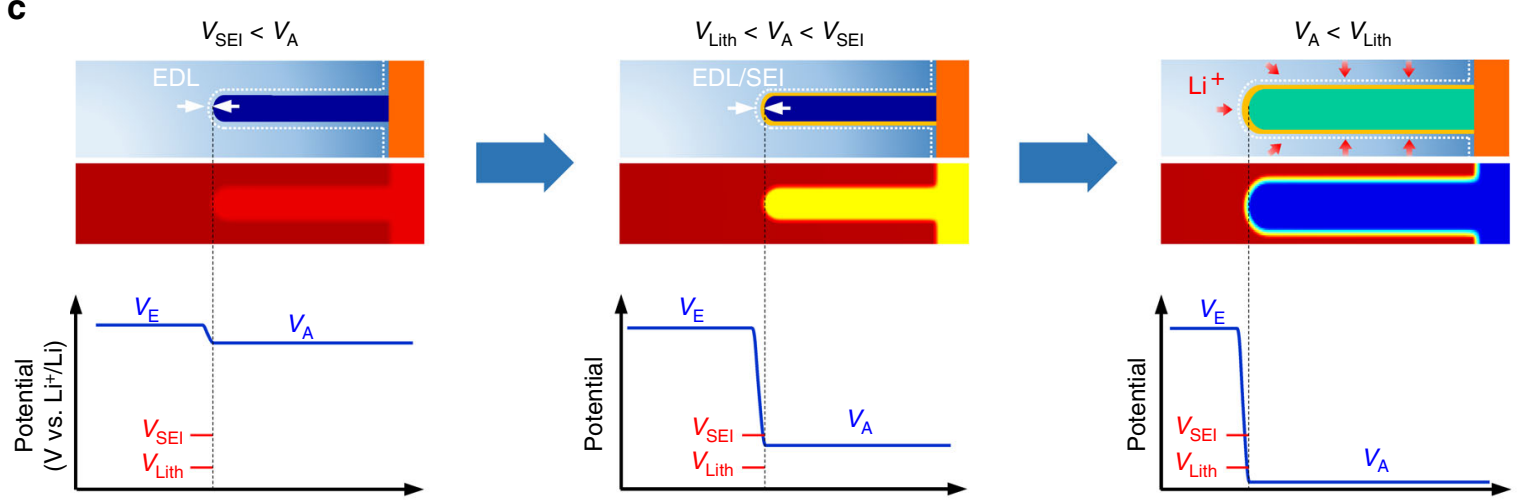

d

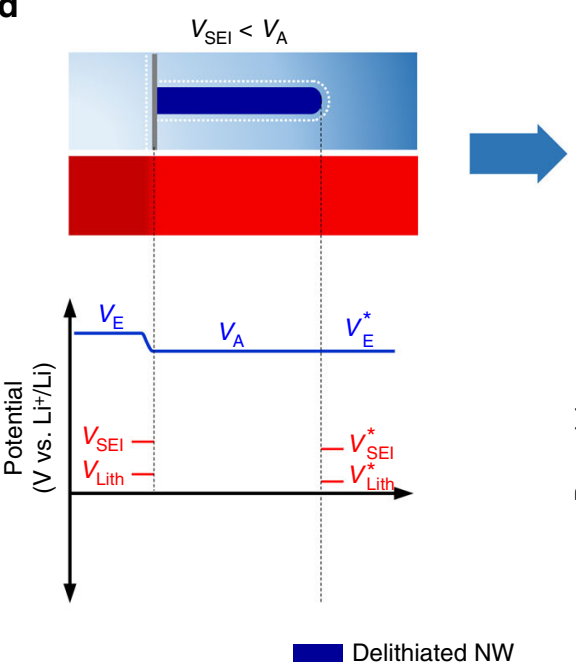

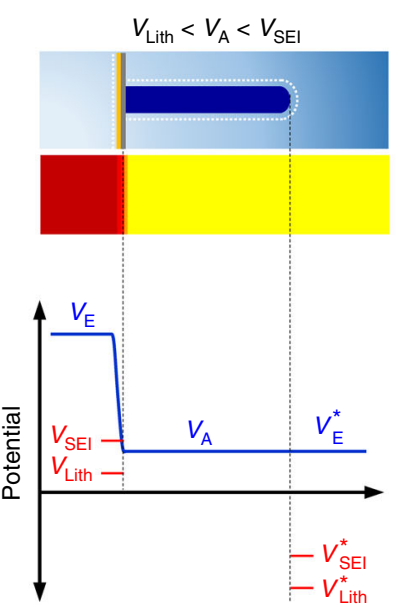

Lithiated NW
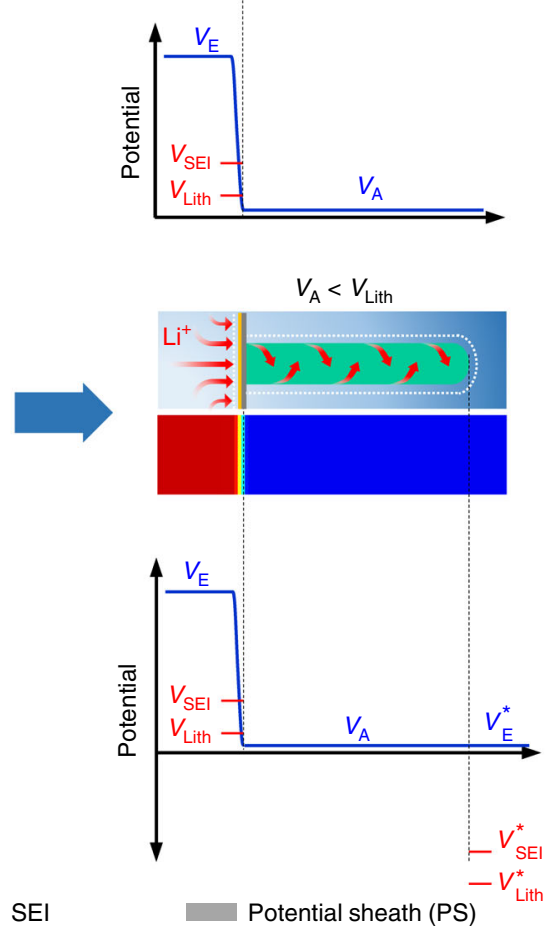

Fig. 1 Schematic and electrical potential profiles of the NW-based anodes. a, b Schematics of a half-cell with a general NW anode on a current collector (a) and a half-cell with an NW anode enclosed by PS (b) paired with counter electrodes. An external load drives the NW anode to become lithiated. c Schematics and corresponding FEA simulation results of electrical potential profiles across the electrolyte and NW anode for the case in $\mathbf{a}$, during the first charging cycle. SEl formation and lithiation start at a potential below the $V_{\text {SEl }}$ and the $V_{\text {Lith, }}$ respectively. $\mathbf{d}$ Schematic and corresponding electrical potential profiles across the outer electrolyte, electrode (PS, NW anode), and inner electrolyte for the case in $\mathbf{b}$ during the first charging. Inside the space enclosed by the PS, the potentials across the electrode and electrolyte became nearly equivalent, thereby suppressing SEl formation on the NW surface and lithiation at the electrolyte and NW interface

develops within the EDL formed on the surface of the graphene PS, while the potential inside the PS remains nearly equivalent to the values of the anode (Fig. 2b and Supplementary Fig. 4 for information about this simulation). It is important to note that our concept can tolerate both anode-electrolyte contact and the existence of nanoscale cracks in the PS (Supplementary Fig. 4b, c). This is different from previous approaches (e.g., yolk-shell structures, in which the internal voids should be kept electrolyte-free by blocking electrolyte permeation with a defectfree sturdy shell); thus, it has advantages in terms of defect tolerance and reliable operation. Alternatively, when the graphene
PS has a macroscopic hole, penetration of an external electric potential through the hole countervails the potential shielding effect (Supplementary Fig. 4d).

NiSiNWs are explored as an active anode material; these NWs are attractive due to their potential for high-capacity and good rate capability, as well as their low-temperature growth in the presence of $\mathrm{Ni}$ and $\mathrm{Si}$ precursors $\left(\sim 450{ }^{\circ} \mathrm{C}\right)^{20}$. NiSi has a gravimetric capacity of $\sim 1300 \mathrm{mAh} \mathrm{g}^{-1,21-23}$ which is lower than that of Si but still much higher than graphite. An additional advantage of $\mathrm{NiSi}$ is related to its metallic conduction, with a typical resistivity of $\sim 10 \mu \Omega \mathrm{cm}$ for single crystals ${ }^{24}$. As 

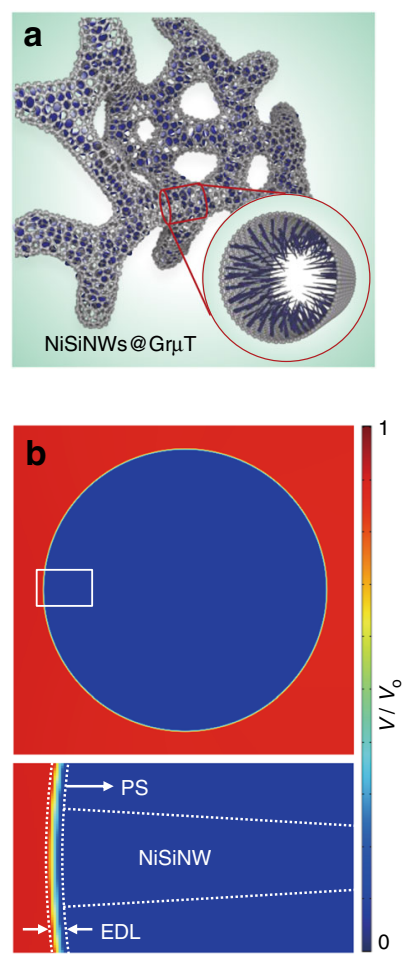
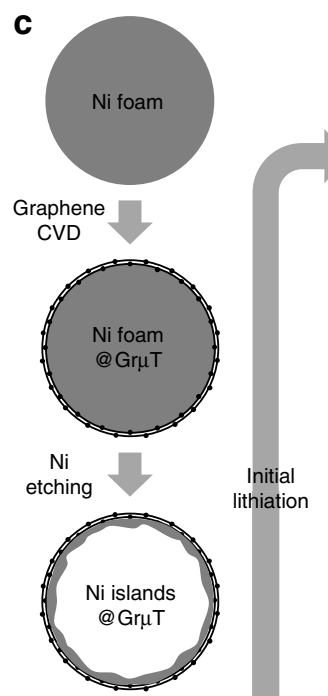

Si CVD

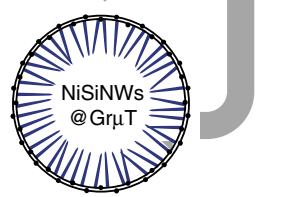

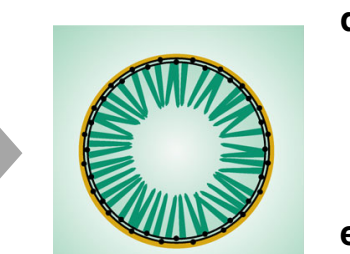
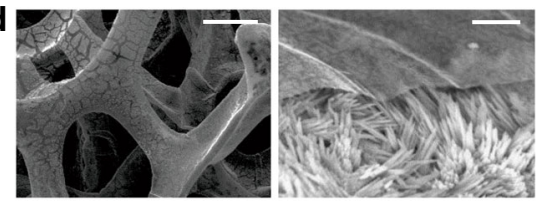

e
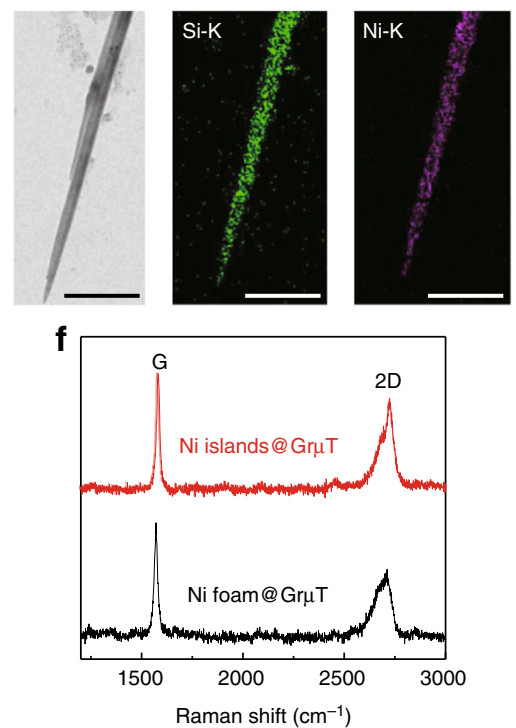

Fig. 2 Fabrication and structural analyses of NiSiNWs@Gr $\mu$ T. a Schematic of NiSiNWs@Gr $\mu$ T. b Simulation of the potential distribution across the NWs@Gr $\mu$ T with an SEI layer on the outer surface of the Gr $\mu \mathrm{T}$. Bottom panel: enlarged images taken from the square in the top panel showing that the formation of the SEI on the outer surface of the Gr $\mu$ T occurred through the development of a rapid potential gradient. A voltage difference of $V_{0}$ was applied between the left-hand electrolyte and Gr $\mu \mathrm{T}$, and the calculated position-dependent potential values were normalized with respect to $V_{\mathrm{o}}$. c Schematic of the fabrication process of NWs@Gr $\mu$ T (left) and lithiation-delithiation cycling (right). SEl formation and Li-ion insertion/extraction only occur around the outer surface of the Gr $\mu$ T. d SEM images of as-fabricated (left) and cross-sectioned (right) NiSiNW@Gr $\mu$ T. Scale bars, $50 \mu \mathrm{m}$ (left) and 2 $\mu \mathrm{m}$ (right). e TEM image of NiSiNW (left) and EDS elemental mapping images of Si (middle) and Ni (right). A Ni to Si atomic ratio of $\sim 1: 1$ was confirmed by EDS composition analysis. Scale bars, $400 \mathrm{~nm}$ (left) and $250 \mathrm{~nm}$ (middle and right). f Raman spectra of the Gr $\mu \mathrm{T}$ before (black) and after (red) core Ni etching

schematically shown in Fig. 2c, the key steps to achieve NiSiNWs@Gr $\mu$ T include (i) chemical vapor deposition (CVD) growth of few-layer graphene on $\mathrm{Ni}$ foam, (ii) partial etching of core $\mathrm{Ni}$ in solution to yield residual $\mathrm{Ni}$ islands on the inner surface of the Gr $\mu \mathrm{T}$, and (iii) CVD growth of NiSiNWs. A detailed description of the overall procedure is given in the Methods and scanning electron microscopy (SEM) images of each step and energy-dispersive X-ray spectroscopy (EDS) elemental mapping images of the broken edges of the NiSiNWs@Gr $\mu$ T are shown in Supplementary Figs. 5, 6, respectively.

The SEM image in the left panel of Fig. $2 \mathrm{~d}$ shows the clean exterior of the final product, which is similar to that of the initial $\mathrm{Ni}$ foam (although some shrinkage can be observed). Since the NiSiNWs@Gr $\mu \mathrm{T}$ is fabricated with coin-shaped Ni, the Gr $\mu \mathrm{T}$ ends remain sealed, as confirmed by SEM images (Supplementary Fig. 5e, f). When the sample was cut after being frozen in liquid nitrogen, very thin graphene tubular walls and a high density of NWs were observed (right panel in Fig. 2d). Typically, the diameter of the Gr $\mu \mathrm{T}$ is in the range of $40-60 \mu \mathrm{m}$, while the diameter and length of NiSiNWs are $\sim 80-150 \mathrm{~nm}$ and $8-10 \mu \mathrm{m}$, respectively. To identify the atomic composition and structure of the NiSiNWs, transmission electron microscopy (TEM) and Xray diffraction (XRD) analyses were performed. The energydispersive X-ray spectroscopy (EDS) composition analysis of a single NW reveals the uniform distribution of $\mathrm{Ni}$ and $\mathrm{Si}$ elements with an atomic ratio of almost 1:1 (Fig. 2e and Supplementary Fig. 7). A lattice-resolved high-resolution TEM image and selected area electron diffraction (SAED) pattern of the NWs also confirm the single-crystal structure of the NiSi core, which coincides with the appearance of XRD peaks of the crystalline $\mathrm{NiSi}$ phase after the CVD growth of NiSiNWs, with a thin amorphous shell (Supplementary Fig. 8). From the TEM images of the fragment separated from the NiSiNWs@Gr $\mu \mathrm{T}$, we found that thin island layers remained on the inside surface of the Gr $\mu \mathrm{T}$ (Supplementary Fig. 9). The EDS analysis confirmed that those islands are amorphous $\mathrm{SiO}_{\mathrm{X}}$ embedded with 5-20-nm-diameter $\mathrm{Ni}$ nanoparticles. In addition, the Raman spectra of the Gr $\mu \mathrm{T}$ collected before and after $\mathrm{Ni}$ etching in Fig. $2 \mathrm{f}$ exhibit characteristic features, with a $2 \mathrm{D}$ to $\mathrm{G}$ band ratio in the range of $0.6-0.8$, which is similar to the fingerprint of few-layer graphene (five to seven layers) grown on $\mathrm{Ni}$ foil (see also Supplementary Fig. 10) ${ }^{25}$. Interestingly, disorder-induced peaks, such as the D peak $\left(\sim 1350 \mathrm{~cm}^{-1}\right)$ corresponding to the presence of disorder in $s p_{2}$-hybridized carbon systems, are difficult to observe in both samples. These results indicate that the Gr $\mu \mathrm{T}$ sustain the original characteristics of few-layer graphene without substantial degradation during Ni etching and NiSiNW growth ${ }^{26}$.

Electrochemical performance of NiSiNWs@GruT anodes. The electrochemical performance of NiSiNWs@Gr $\mu \mathrm{T}$ anodes is tested in a CR2032 coin-type cell using Li-metal foil as a counter electrode. Figure 3 a shows the evolution of the cyclic voltammetry (CV) profiles for the NiSiNWs@Gr $\mu \mathrm{T}$ (1st, 3rd, 5th, and 7th scans). The peaks at 0.1 and $0.22 \mathrm{~V}$ in the cathodic scan and at $0.27 \mathrm{~V}$ in the anodic scan correspond to lithiation and delithiation of NiSiNWs, respectively, and exhibit increasing current density after the scans. In contrast, other peaks at $1.4 \mathrm{~V}$ in the cathodic 

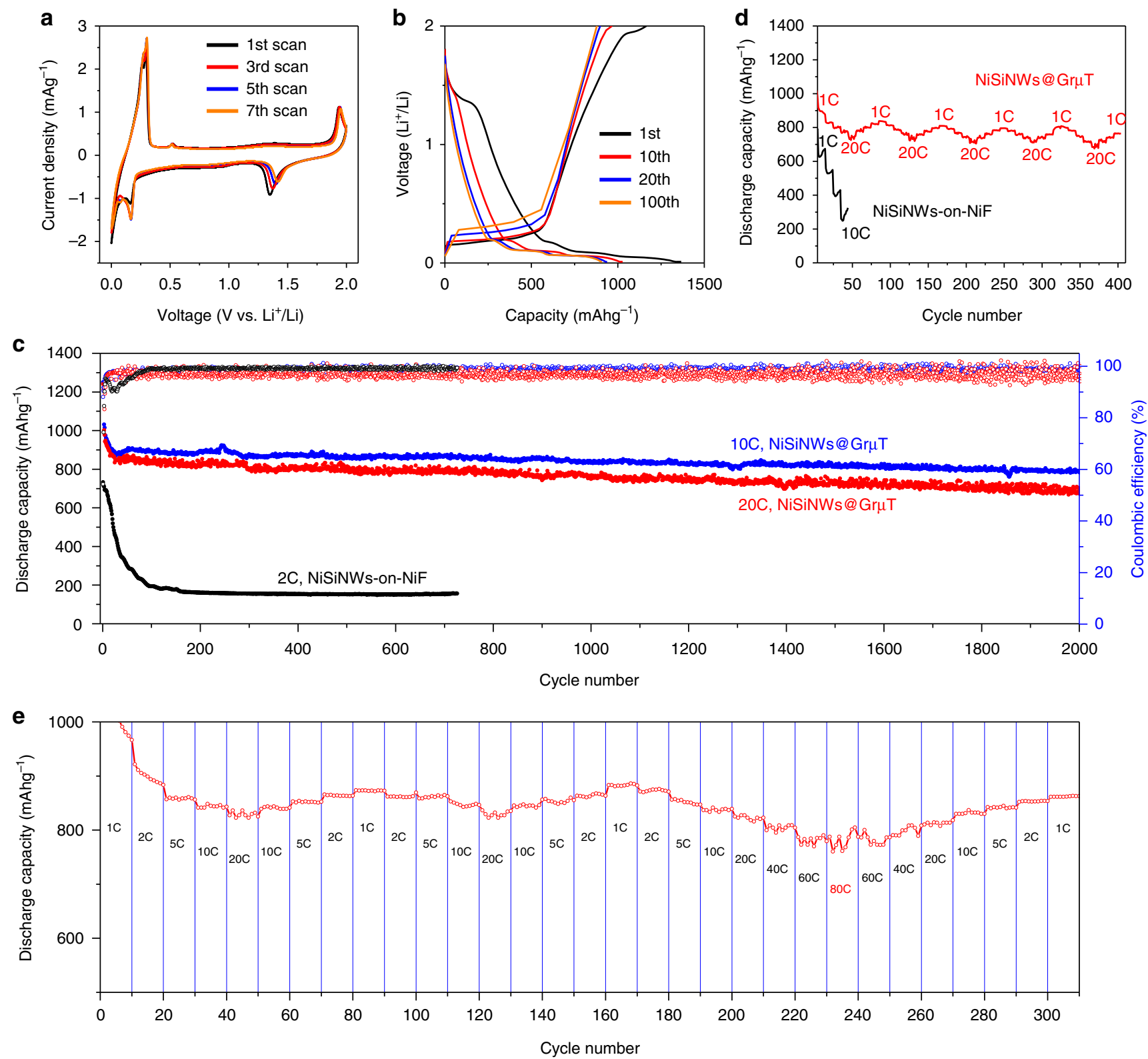

Fig. 3 Electrochemical analysis of NiSiNWs@Gr $\mu \mathrm{T}$. a CV profiles of NiSiNWs@Gr $\mu$ T during sweeps over the range from 2.0 to $0.0 \mathrm{~V}$ vs. Li/Li+ at a rate of $0.1 \mathrm{mV} \mathrm{s}^{-1}$. b Galvanostatic charge-discharge profiles of NiSiNWs@Gr $\mu$ T for the 1st, 10th, 20th, and 100th cycles. c Capacity of NiSiNWs@Gr $\mu$ T cycled at the discharging rate while undergoing stepwise changes from $1 \mathrm{C}$ to $20 \mathrm{C}$. The NiSiNWs-on-NiF is used for comparison (black). $\mathbf{d}$ Discharge capacity (solid circles) and coulombic efficiency (open circles) of NiSiNWs@Gr $\mu$ T at 10C (black) and 20C (red) for 2000 cycles. The same plots of NiSiNWs-on-NiF at 2C (blue). e Rate capability test of NiSiNWs@Gr $\mu$ T with various cycling rates from $1 \mathrm{C}$ to $80 \mathrm{C}$. All tests were performed using 1.0 M LiPF 6 in EC/DEC (1:1) as the electrolyte, in the potential window between $0.0 \mathrm{~V}$ and $2.0 \mathrm{~V}$ ( $\mathrm{vs} . \mathrm{Li}_{\mathrm{Li}}{ }^{+}$). All the capacities are represented based on the weight of $\mathrm{NiSiNWs}$

scan and at $1.9 \mathrm{~V}$ in the anodic scan diminish gradually during the scans. We can also observe plateaus at 1.4 and $1.9 \mathrm{~V}$ in the galvanostatic charge-discharge (GCD) profiles, displaying similar features in the charging and discharging stages, respectively, and almost disappear after 10-20 cycles (Fig. 3b). The nearly identical behaviors were observed from the nickel sulfide anode ${ }^{27,28}$ and the samples that underwent sulfuric acid treatment (Supplementary Figs. 11, 12) ${ }^{29}$. Thus, we conclude that the plateaus/ peaks at 1.4 and $1.9 \mathrm{~V}$ are associated with the lithiation and delithiation of nickel sulfide residues that were developed during the nickel etching process using sulfuric acid, respectively. It is noteworthy that the lithiation plateaus disappeared rapidly during the initial several cycles (Fig. 3b). Since the delithiation potential of nickel sulfide shifted toward higher voltage with cycles and exceeded the upper limit of the voltage window $(2 \mathrm{~V})^{28}$, the more lithiated nickel sulfide could not return to a delithiated state with cycles. This mechanism is closely linked to the rapid capacity fading that tends to occur in NiSiNWs@Gr $\mu$ T anodes in the early stage of cycles (see the red and blue plots in Fig. $3 c-e)^{30}$. Nevertheless, once the anodes were stabilized, there was little change in the charge/discharge profile after 100 cycles at $10 \mathrm{C}$, and the charge/discharge capacities were maintained above $900 \mathrm{mAh} \mathrm{g}^{-1}$.

To further evaluate the performance of NiSiNWs@GruT anodes, charge/discharge cycling tests were performed at $10 \mathrm{C}$ and $20 \mathrm{C}$ for more than 2000 cycles. The resulting discharge capacity and Columbic efficiency are plotted as a function of the cycle number in Fig. 3c. For comparison, the anode with 
NiSiNWs-on-Ni foam (NiSiNWs-on-NiF) was also tested at a much slower cycling rate of $2 \mathrm{C}$ (see Supplementary Fig. 13 for preparation information about this sample). While the anode with NiSiNWs-on-NiF showed significant capacity fading, with 33\% capacity retention after 100 cycles, the NiSiNWs@GruT anodes showed superior performance and cycling stability. With the exception of capacity fading during the initial formation cycles (up to the 20th cycle) ${ }^{30}$, the capacity retentions of the NiSiNWs@Gr $\mu$ T anodes are as high as $90 \%$ at $10 \mathrm{C}$ and $84 \%$ at 20C after 2000 cycles. The comparison of the real capacities of

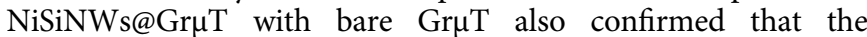
contribution of graphene to the total capacity was $<2 \%$ of the total capacity (Supplementary Fig. 14).

Given that changes in the charging/discharging rates can accelerate structural failure of the electrode materials, rate capability is another important consideration for high-power operation. We investigated the rate capabilities of anodes made from NiSiNWs@Gr $\mu \mathrm{T}$ and NiSiNWs-on-NiF under a series of stepwise increases and decreases in the discharging rate from $1 \mathrm{C}$ to $20 \mathrm{C}(1 \mathrm{C}, 2 \mathrm{C}, 5 \mathrm{C}, 10 \mathrm{C}$, and $20 \mathrm{C} ; 10$ cycles at each rate). As shown in Fig. 3d, even when the charging rate increased 20 -fold from 1C to 20C, the NiSiNWs@Gr $\mu$ T anode maintained $91 \%$ of its highest capacity at 1C (except for the initial stabilization phase). In contrast, abrupt decay of the charge capacity was observed in the NiSiNWs-on-NiF anode with increasing rates and cycles so that the capacity difference between the anodes became more pronounced with a greater number of cycles. The NiSiNWson- $\mathrm{NiF}$ anode retained a capacity of $270 \mathrm{mAh} \mathrm{g}^{-1}$ at $10 \mathrm{C}$, which is $<34 \%$ of the capacity of the NiSiNWs@Gr $\mu$ T. More importantly, NiSiNWs@Gr $\mu \mathrm{T}$ anodes demonstrate excellent rate capability under more severe cycling conditions, with a stepwise increase and decrease in the rate from $1 \mathrm{C}$ to $20 \mathrm{C}$ and from $1 \mathrm{C}$ to $80 \mathrm{C}$, respectively (Fig. 3e). The anode maintained a capacity above $800 \mathrm{mAh} \mathrm{g}^{-1}$ at $40 \mathrm{C}$ and above $780 \mathrm{mAhg}^{-1}$ at $80 \mathrm{C}$, retaining 90 and $88 \%$ of the capacity at $1 \mathrm{C}$, respectively. The comparison of these results with state-of-the-art LIB anodes in the literature illustrates the substantial advantages of the NiSiNWs@GruT anode (Supplementary Fig. 15). Importantly, only a few reports have addressed the cycling performance of anodes under extreme cycling conditions and charge/discharge rates higher than $10 \mathrm{C}^{31}$. To the best of our knowledge, there is no report demonstrating a capacity higher than $780 \mathrm{mAh} \mathrm{g}^{-1}$ at speeds higher than $80 \mathrm{C}$.

\section{Discussion}

We ascribe the unprecedentedly superior cycling performance of the NiSiNWs@Gr $\mu \mathrm{T}$ anode to the synergetic advantages of the Gr $\mu \mathrm{T}$ and NiSiNWs; an SEI layer develops only on the outer surface of the Gr $\mu$ T-based PS, and the SEI-free NiSiNWs allow fast electron/ionic transport and reversible volumetric change. SEM images of the NiSiNWs@GruT taken after cycling confirmed that the NiSiNWs maintained most of their original shape and size, although they did show a roughened surface (Fig. $4 \mathrm{a}, \mathrm{b}$ ). The selective formation of an SEI layer on the outer surface of the $\mathrm{Gr} \mu \mathrm{T}$, which agrees with our hypothesis, was also confirmed (Fig. 4a, right panel). The comparison of the lithiated and delithiated NiSiNWs showed that the diameter increased by an average of $190 \%$, which is comparable to the theoretical expectation (Fig. 4b) ${ }^{32}$. TEM images of a single delithiated NW, detached from the Gr $\mathrm{TT}$, showed a polycrystalline core and amorphous shell (Fig. 4c); these can be assigned to $\mathrm{NiSi}$ and $\mathrm{SiO} x$ $(\mathrm{NiO} x)$, respectively, based on EDS elemental analysis (Fig. $4 \mathrm{~d}$ and Supplementary Fig. 16). Importantly, the P and F associated with the SEI layer component are hardly detected in the NiSiNWs ${ }^{33}$. In contrast, the EDS peaks corresponding to $\mathrm{F}$ and $\mathrm{P}$

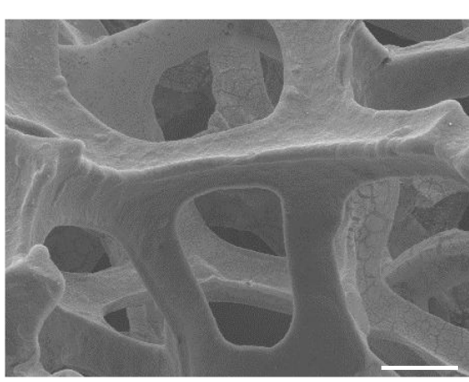

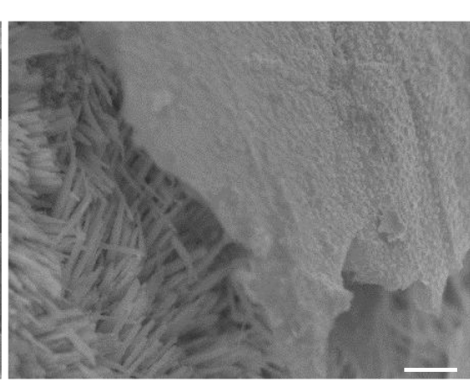

b

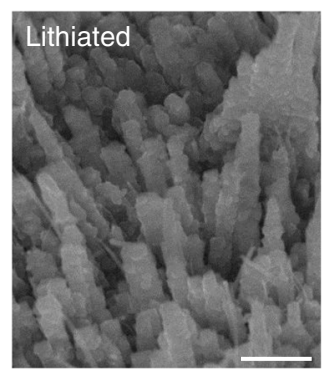

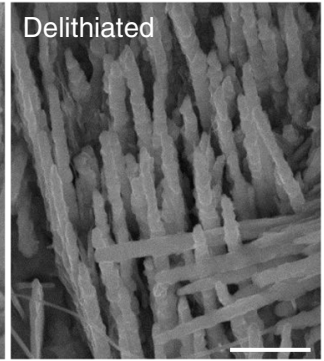

C

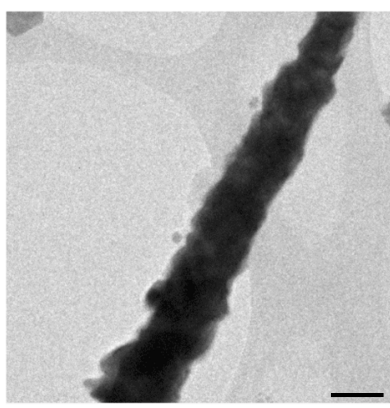

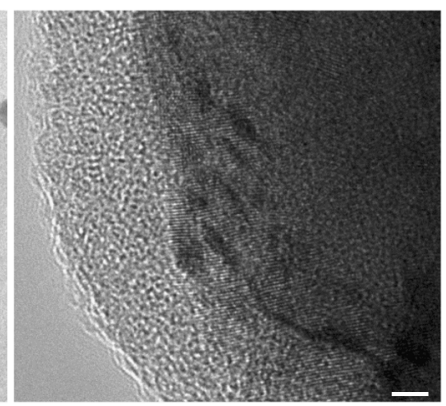

d

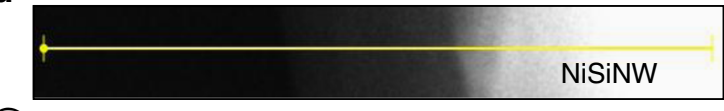

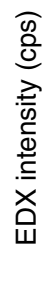

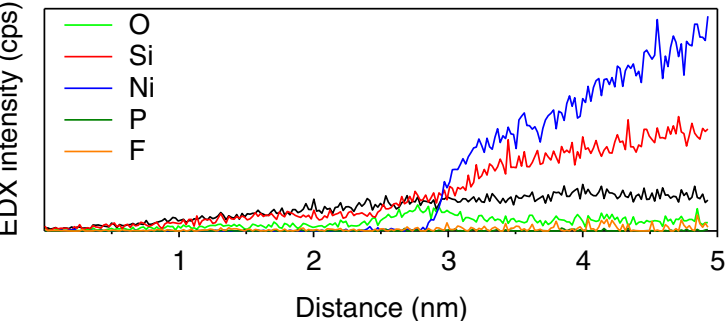

Fig. 4 Morphology and SEl formation of NiSiNWs@Gr $\mu$ T after cycling. a SEM image of delithiated NiSiNWs@Gr $\mu$ T after cycling. Close-up SEM image of the intentionally-fractured part of the NiSiNWs@Gr $\mu$ T (right), which shows near SEl-free NW surfaces inside the Gr $\mu$ T covered with a thick SEl layer. Scale bars, $100 \mu \mathrm{m}$ (left) and $2 \mu \mathrm{m}$ (right). b SEM images of the lithiated (left) and delithiated (right) NWs inside the Gr $\mu$ T (with the same magnification). The diameter of the NWs increases by an average of $190 \%$ after lithiation. Scale bars, $1 \mu \mathrm{m}$. c TEM image of delithiated NiSiNW after cycling (left) and enlarged HR-TEM images taken from the square in the left panel, showing the polycrystalline NiSi core covered with a 5-nm-thick oxide amorphous shell (right). $100 \mathrm{~nm}$ (left) and $2 \mathrm{~nm}$ (right). d EDS element analysis of delithiated NiSiNW, taken along the solid line in the upper panel of the dark-field image of the NiSiNW and oxide region. SEl-related components (e.g., P and F) were not detected 
elements are clearly seen for the outer surfaces of the Gr $\mu \mathrm{T}$ (Supplementary Fig. 17a, b). These results support the validity of our approach, which is based on electric potential control to influence SEI development. In the case of the NiSiNWs-on-NiF anode, all of the NWs are embedded with a thick SEI layer after 100 cycles; the individual NW morphology can be identified only after removing the SEI with acetonitrile (Supplementary Fig. 17c and 18$)^{34}$. This result is also coincident with FEA modeling that shows the existence of an abrupt potential drop on the surface of the NiSiNWs (Supplementary Fig. 4e). Although we cannot exclude the contribution of residual Ni nanoparticles (Supplementary Fig. 9) to the improved rate performance, our in-depth analysis and consideration illustrate that the 'potential shielding' effect has a major role in achieving high rate capability.

Despite the excellent cyclability of the NiSiNWs@GruT, there were large fluctuations of capacity and coulombic efficiency at higher rates (>20C); the coulombic efficiency occasionally exceeded $100 \%$ (Fig. 3c, e). We attribute this to the limited Li diffusion in the NWs during ultrafast cycling, which prevents complete lithiation/delithiation ${ }^{35}$. For instance, the residual $\mathrm{Li}$ in the delithiated state would reduce the charge capacity in the next cycle, whereas these Li remnants can be released during the next discharging stage, causing the discharge capacity to exceed the charge capacity. For Li diffusion, the NWs inside the PS would have a disadvantage considering that the potential gradient that drives the lithiation does not develop at the NW and electrolyte interface. However, previous in situ TEM studies showed fast lithiation kinetics in individual NWs, in which $\mathrm{Li}$ insertion occurred at one end of the NWs and was followed by Li transport along the axial direction, similar to our situation. In some cases, the overall lithiation rate was not axial transport limited, but rather determined by the radial insertion of $\mathrm{Li}$ into the $\mathrm{NW}$ core $^{36}$. This is due to the much faster Li transport along the surface than that in the bulk, enabling ultrafast lithiation with an axial speed up to $\sim 213 \mathrm{~nm} \mathrm{~s}^{-1}$ for highly conducting SiNWs ${ }^{37}$. In this regard, we conclude that the Li diffusion in our NW anode does not critically limit the cycling capacity even at higher rate (e.g., the axial lithiation speed of $200 \mathrm{~nm} \mathrm{~s}^{-1}$ can fully lithiate $\sim 8$ $\mu \mathrm{m}$-long SiNWs at $80 \mathrm{C}$ ). Nonetheless, further study is needed to elucidate the lithiation kinetics in our system.

In summary, we suggest a new strategy for inhibiting SEI formation on the anode surface through engineering the electric potential using an atomically thin and ion-permeable PS. The NiSiNWs@GruT were tested as a proof-of-concept for the proposed strategy and demonstrated excellent performance during 2000 cycles at 20C with a high specific capacity of $702 \mathrm{mAh} \mathrm{g}^{-1}$, corresponding to $84 \%$ of the initial capacity. Moreover, the NiSiNWs@Gr $\mu$ T anodes showed superior rate capabilities with a capacity retention higher than $88 \%$ at $80 \mathrm{C}$ (vs. the capacity at $1 \mathrm{C}$ ). Because our approach is based on general electrochemistry, it is applicable to other materials (e.g., Si-based anode) to further improve their capacity. It can also be used with a variety of electrochemical devices and components.

\section{Methods}

Fabrication of NiSiNWs@Gr $\boldsymbol{\mu T}$. The NiSiNWs@Gr $\mu$ T were fabricated by growing NiSiNWs selectively inside the graphene-based 3D micro-tubular structures. First, high-purity Ni foam (1 mm in thickness) was purchased from MTI Korea and cut into coin-like shapes using a punching machine to facilitate cell assembly. Next, the $\mathrm{Ni}$ foam was loaded in the CVD reactor chamber to synthesize multilayer graphene on the surface of the $\mathrm{Ni}$ foam. The reactor was evacuated below $5 \times 10^{-3}$ torr and heated to $1000{ }^{\circ} \mathrm{C}$. After annealing the $\mathrm{Ni}$ foam for $30 \mathrm{~min}$ under $\mathrm{H}_{2} / \mathrm{Ar}$ atmospheric pressure, a gas mixture containing $30 \% \mathrm{CH}_{4}$ diluted in $\mathrm{H}_{2}$ and $\mathrm{Ar}$ was introduced into the reactor to initiate graphene growth. After $2 \mathrm{~min}$ of exposure to the precursor gas mixture, the reactor was evacuated again and immediately cooled to room temperature. The color change of the Ni foam after uniform coating with multilayer graphene was recognizable to the naked eye. The sample was treated with ethanol (EtOH) and then immersed into a Ni etchant (UN2796 sulfuric acid, Transene
Company, Inc.), which acts as a surfactant to prevent the formation of air bubbles inside the sample. Samples were immersed in the $\mathrm{Ni}$ etchant for $2.5 \mathrm{~h}$, until only a small amount of $\mathrm{Ni}\left(0.45 \mathrm{mg} \mathrm{cm}^{-2}\right)$ remained inside the Gr $\mu \mathrm{T}$. The sample was then rinsed three times with deionized water and dried fully in an oven $\left(70{ }^{\circ} \mathrm{C}\right)$. After complete drying, the sample was loaded into a silicon CVD reactor to grow NiSiNWs The growth of NiSiNWs occurs selectively inside the Gr $\mu \mathrm{T}$ in the presence of the residual $\mathrm{Ni}$ and $\mathrm{Si}$ precursors of $\mathrm{SiH}_{4}$. During NiSiNW growth, a gas mixture of $10 \%$ $\mathrm{SiH}_{4}$ diluted with $\mathrm{H}_{2}$ was introduced at a flow rate of $50 \mathrm{sccm}$, and the reactor temperature and pressure were maintained at $\sim 460^{\circ} \mathrm{C}$ and 20 torr, respectively. The typical growth time was $20 \mathrm{~min}$. A reference anode consisting of NiSiNWs directly grown on $\mathrm{Ni}$ foam was also prepared using identical Si CVD processing conditions.

Structural characterization. For structural and elemental analyses of the samples, FE-SEM (JEOL JSM-7600 with $15 \mathrm{kV}$ ), TEM equipped with EDS (JEOL JEM2100F, Cs corrector), Raman spectroscopy (MonoRa 750i, Dongwoo Optron Co.), and XRD (Rigaku D/MAX RINT-2000) were used. For cross-sectional SEM measurements, the NiSiNWs@Gr $\mu$ T samples were cut after being frozen in liquid nitrogen. After cycling, the NiSiNWs@Gr $\mu$ T anode was rinsed three times with the $\mathrm{EC} / \mathrm{EDC}$ solvent and then dried in a glove box.

Electrochemical characterization. NiSiNWs were assembled into coin-type half cells (CR2032) in an argon-filled glove box. Li metal was used as a counter electrode, and 1.0 $\mathrm{M} \mathrm{LiPF}_{6}$ in ethylene carbonate/diethylene carbonate (EC/DEC, 1:1 vol.\%) was used to fill the half cells. CV profiles were determined using an electrochemical workstation (IVIUM-n-STAT). CV was performed at a scan rate of $0.1 \mathrm{mV} \mathrm{s}^{-1}$ between 0 and $2 \mathrm{~V}$. All of the GCD profiles and rate/cycling performances of the coin-type cells were tested using a TOSCAT 3000 battery tester (TOSCAT 3000, Japan) between 0.01 and $2 \mathrm{~V}$.

Data availability. The authors declare that all the relevant data are available within the paper and its Supplementary Information file or from the corresponding author on reasonable request.

Received: 6 March 2018 Accepted: 8 August 2018

Published online: 27 August 2018

\section{References}

1. Armand, M. \& Tarascon, J. M. Building better batteries. Nature 451, 652-657 (2008).

2. Goodenough, J. B. \& Kim, Y. Challenges for rechargeable Li batteries. Chem Mater. 22, 587-603 (2010).

3. Chan, C. K. et al. High-performance lithium battery anodes using silicon nanowires. Nat. Nanotechnol. 3, 31-35 (2008).

4. Xia, F. et al. Facile synthesis of free-standing silicon membranes with threedimensional nanoarchitecture for anodes of lithium ion batteries. Nano Lett. 13, 3340-3346 (2013).

5. Song, T. et al. Arrays of sealed silicon nanotubes as anodes for lithium ion batteries. Nano Lett. 10, 1710-1716 (2010).

6. Mo, R. W., Rooney, D., Sun, K. N. \& Yang, H. Y. 3D nitrogen-doped graphene foam with encapsulated germanium/nitrogen-doped graphene yolk-shell nanoarchitecture for high-performance flexible Li-ion battery. Nat. Commun. 8, 13949 (2017).

7. Qin, J. et al. Graphene networks anchored with Sn@graphene as lithium ion battery anode. ACS Nano 8, 1728-1738 (2014).

8. Lee, S. W., McDowell, M. T., Berla, L. A., Nix, W. D. \& Cui, Y. Fracture of crystalline silicon nanopillars during electrochemical lithium insertion. Proc. Natl Acad. Sci. USA 109, 4080-4085 (2012).

9. Sun, Y. M., Liu, N. A. \& Cui, Y. Promises and challenges of nanomaterials for lithium-based rechargeable batteries. Nat. Energy 1, 16071 (2016).

10. Park, M. H. et al. Silicon nanotube battery anodes. Nano Lett. 9, 3844-3847 (2009).

11. Xia, F. et al. Graphene as an interfacial layer for improving cycling performance of Si nanowires in lithium-ion batteries. Nano Lett. 15, 6658-6664 (2015)

12. $\mathrm{Wu}, \mathrm{H}$. et al. Stable cycling of double-walled silicon nanotube battery anodes through solid-electrolyte interphase control. Nat. Nanotechnol. 7, 309-314 (2012).

13. Liu, N. et al. A pomegranate-inspired nanoscale design for large-volumechange lithium battery anodes. Nat. Nanotechnol. 9, 187-192 (2014).

14. Li, Y. S., Leung, K. \& Qi, Y. Computational exploration of the Lielectrodelelectrolyte interface in the presence of a nanometer thick solidelectrolyte interphase layer. Acc. Chem. Res. 49, 2363-2370 (2016).

15. Soto, F. A., Ma, Y. G., de la Hoz, J. M. M., Seminario, J. M. \& Balbuena, P. B. Formation and growth mechanisms of solid-electrolyte lnterphase layers in rechargeable batteries. Chem. Mater. 27, 7990-8000 (2015). 
16. Tarascon, J. M. \& Armand, M. Issues and challenges facing rechargeable lithium batteries. Nature 414, 359-367 (2001).

17. Bignozzi, C. A. et al. Nanostructured photoelectrodes based on WO3: applications to photooxidation of aqueous electrolytes. Chem. Soc. Rev. 42, 2228-2246 (2013).

18. Cheng, C. et al. Ion transport in complex layered graphene-based membranes with tuneable interlayer spacing. Sci. Adv. 2, 1501272 (2016).

19. Yoon, T., Mun, J. H., Cho, B. J. \& Kim, T. S. Penetration and lateral diffusion characteristics of polycrystalline graphene barriers. Nanoscale 6, 151-156 (2014).

20. Kang, K., Kim, S. K., Kim, C. J. \& Jo, M. H. The role of NiOx overlayers on spontaneous growth of NiSix nanowires from Ni seed layers. Nano Lett. 8, 431-436 (2008).

21. Wang, G. X. et al. Nanocrystalline NiSi alloy as an anode material for lithiumion batteries. J. Alloy. Compd. 306, 249-252 (2000).

22. Zhou, Y. N. et al. Nanostructured NiSi thin films as a new anode material for lithium ion batteries. Electrochem. Commun. 13, 546-549 (2011).

23. $\mathrm{Li}, \mathrm{F}$. et al. In situ coating of $\mathrm{NiO}$ on $\mathrm{Ni}$-silicide nanowires with roughened surfaces for improved electrochemical energy storage. J. Mater. Chem. A 2, 9156-9163 (2014).

24. Kim, J. \& Anderson, W. A. Direct electrical measurement of the selfassembled nickel silicide nanowire. Nano. Lett. 6, 1356-1359 (2006).

25. Chen, Z. P. et al. Three-dimensional flexible and conductive interconnected graphene networks grown by chemical vapour deposition. Nat. Mater. 10, 424-428 (2011).

26. Lee, D. H. et al. Engineering electronic properties of graphene by coupling with Si-rich, two-dimensional islands. ACS Nano 7, 301-307 (2013).

27. Lai, C. H. et al. Oriented growth of large-scale nickel sulfide nanowire arrays via a general solution route for lithium-ion battery cathode applications. J. Mater. Chem. 19, 7277-7283 (2009).

28. Mahmood, N., Zhang, C. Z. \& Hou, Y. L. Nickel sulfide/nitrogen-doped graphene composites: phase-controlled synthesis and high performance anode materials for lithium ion batteries. Small 9, 1321-1328 (2013).

29. Kish, J. R., Ives, M. B. \& Rodda, J. R. Corrosion mechanism of nickel in hot, concentrated $\mathrm{H}_{2} \mathrm{SO}_{4}$. J. Electrochem. Soc. 147, 3637-3646 (2000).

30. An, S. J., Li, J. L. \& Wood, D. L. Fast formation cycling for lithium ion batteries (vol 342, pg 846, 2017). J. Power Sources 350, 152-152 (2017).

31. Liu, J., Kopold, P., van Aken, P. A., Maier, J. \& Yu, Y. Energy storage materials from nature through nanotechnology: a sustainable route from reed plants to a silicon anode for lithium-ion batteries. Angew. Chem. Int. Ed. 54, 9632-9636 (2015).

32. Lund, I. N., Lee, J. H., Efstathiadis, H., Haldar, P. \& Geer, R. E. Influence of catalyst layer thickness on the growth of nickel silicide nanowires and its application for Li-ion batteries. J. Power Sources 246, 117-123 (2014)

33. Nguyen, C. C., Yoon, T., Seo, D. M., Guduru, P. \& Lucht, B. L. Systematic investigation of binders for silicon anodes: interactions of binder with silicon particles and electrolytes and effects of binders on solid electrolyte interphase formation. ACS Appl. Mater. Interfaces 8, 12211-12220 (2016).

34. Wu, H. et al. Engineering empty space between Si nanoparticles for lithiumion battery anodes. Nano Lett. 12, 904-909 (2012).

35. Liu, X. H. et al. Self-limiting lithiation in silicon nanowires. ACS Nano 7 , 1495-1503 (2013).
36. Liu, X. H. et al. Anisotropic swelling and fracture of silicon nanowires during lithiation. Nano Lett. 11, 3312-3318 (2011).

37. Liu, X. H. et al. Ultrafast electrochemical lithiation of individual Si nanowire anodes. Nano Lett. 11, 2251-2258 (2011).

\section{Acknowledgements}

This work was supported by the National Research Foundation of Korea (NRF) funded by the Ministry of Science, ICT and Future Planning (MSIP) of Korea (Nos. 2018R1A2B2006410, 2016K1A4A3914691, and 2009-0082580) and by the Korea Institute of Energy Technology Evaluation and Planning (KETEP), granted financial resources from the Ministry of Trade, Industry \& Energy, Republic of Korea (Nos. 20174030201750 and 20168510050080).

\section{Author contributions}

W.J.C. and W.I.P. conceived the concept and designed the experiments. W.J.C. performed the synthesis and electrochemical measurements. W.J.C., S.H.K., J.H., J.C., and W.I.P. performed electrochemical analysis of the results and set up the modeling. D.W Y., S.S.K., J.T.K., W.W.L., S.S.K., J.H.L., H.P., T.S.S., I.-H.L., and D.W. participated in materials synthesis, characterizations, electrochemical measurements, and result analysis. W.J.C. and W.I.P. co-wrote the paper. All authors discussed the results and commented on the manuscript.

\section{Additional information}

Supplementary Information accompanies this paper at https://doi.org/10.1038/s41467018-05986-9.

Competing interests: The authors declare no competing interests.

Reprints and permission information is available online at http://npg.nature.com/ reprintsandpermissions/

Publisher's note: Springer Nature remains neutral with regard to jurisdictional claims in published maps and institutional affiliations.

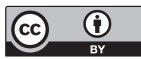

Open Access This article is licensed under a Creative Commons Attribution 4.0 International License, which permits use, sharing, adaptation, distribution and reproduction in any medium or format, as long as you give appropriate credit to the original author(s) and the source, provide a link to the Creative Commons license, and indicate if changes were made. The images or other third party material in this article are included in the article's Creative Commons license, unless indicated otherwise in a credit line to the material. If material is not included in the article's Creative Commons license and your intended use is not permitted by statutory regulation or exceeds the permitted use, you will need to obtain permission directly from the copyright holder. To view a copy of this license, visit http://creativecommons.org/ licenses/by/4.0/.

(c) The Author(s) 2018 